\title{
Impacto de un sitio de disposición final de residuos sólidos en la salud respiratoria de los adultos mayores
}

\author{
Paola Andrea Filigrana, Olga Lucía Gómez, Fabián Méndez \\ Grupo de Epidemiología y Salud Poblacional (sic.), Escuela de Salud Pública, Universidad del Valle, \\ Cali, Colombia
}

Introducción. El objetivo de este estudio fue evaluar el impacto de un sitio de disposición final de residuos sólidos sobre la salud respiratoria de adultos mayores de 50 años, residentes en su área de influencia.

Materiales y métodos. Se llevó a cabo un estudio de cohortes que incluyó un grupo de 313 adultos mayores de 50 años expuestos al Botadero de Navarro y otro de 359 no expuestos, seguidos durante seis meses, para comparar la incidencia mensual de síntomas respiratorios y alteraciones en el flujo espiratorio pico.

Resultados. Después de ajustar por edad, sexo, escolaridad, humedad, afiliación a salud, enfermedades concomitantes, tabaquismo y exposición ocupacional previa, la exposición al Botadero de Navarro se asoció con la presencia de uno o más síntomas respiratorios $\left(O R=1,25 ; I_{95 \%}: 1,01-1,56\right)$, síntomas respiratorios moderados $\left(\mathrm{OR}=1,28 ; \mathrm{IC}_{95 \%}: 0,98-1,66\right)$, pero no se encontró asociación con síntomas respiratorios graves $\left(O R=1,16 ; I_{95 \%}: 0,89-1,50\right)$. Se evidenció una mayor recurrencia de síntomas en los adultos de la zona expuesta (coeficiente de correlación=0,29, $I C_{95 \%}: 0,23-0,33, p=0,000$ ). En los adultos expuestos al Botadero de Navarro, se encontró en la función pulmonar una reducción significativa del flujo espiratorio pico (-15,19 L/minuto; $\left.\mathrm{IC}_{95 \%}:-30,16-0,22\right)$.

Conclusión. Este estudio muestra que, independientemente de los potenciales factores de confusión, la salud respiratoria de los adultos mayores de 50 años expuestos al Botadero de Navarro está afectada con una mayor presencia y cronicidad de síntomas respiratorios moderados y una disminución de la función pulmonar.

Palabras clave: eliminación de residuos sólidos, contaminación ambiental, botaderos a cielo abierto, sistema respiratorio, anciano.

\section{Impact of a waste disposal site on adult respiratory health}

Introduction. This study evaluated the impact of the presence of a dump on respiratory health in adults older than 50 years of age, living in its area of influence.

Materials and methods. A cohort study was conducted with 313 adults older than 50 years of age living near the Navarro Dump (Cali, Colombia) and compared with 359 adults in an unexposed area. Each was examined monthly during a 6 month period to compare the incidence of respiratory symptoms and alterations in peak expiratory flow.

Results. Exposure to the Navarro Dump was associated with occurrence of respiratory symptoms (odds ratio $=1.25,95 \%$ C.I. 1.01-1.56), in particular with mild respiratory symptoms (odds ratio=1.28, 95\% C.I. 0.98-1.66). This result was obtained after adjusting for age, sex, education level, humidity, health insurance, comorbidity, smoking and previous occupational exposure. A higher recurrence of symptoms was found in adults in the area of exposure (correlation coefficient $=0.29,95 \%$ C.I. $0.23-0.33$, $p<0.001)$ and a significant reduction in the lung function defined by peak expiratory flow; the average decrease was $15.2 \mathrm{~L} / \mathrm{min}$ (95\% C.I. -30.2 to +0.22$)$.

Conclusion. Independent of potential confounding factors, the respiratory health of adults exposed to the Navarro Dump was affected and exhibited an increased occurrence and recurrence of mild respiratory symptoms as well as decreased lung function.

Key words: Refuse disposal, environmental pollution, open dumps, respiratory system, aged.

\section{Correspondencia:}

Paola Andrea Filigrana, Escuela de Salud Pública, Universidad del Valle, Calle 4B № 36-140, edificio 118, Cali, Colombia

Teléfono: (572) 554 2476; fax: (572) 5570425

pafiligrana@grupogesp.org

Recibido: 18/05/11; aceptado:28/04/11
Los rellenos sanitarios son sitios para la disposición de los residuos sólidos, diseñados con el fin de hacer el tratamiento final de basuras y minimizar los impactos en el ambiente; sin embargo, especialmente cuando no son técnicamente bien manejados, emiten contaminantes que pueden 
causar daños en la salud humana (1-4). Aunque los estudios sobre los potenciales efectos de los rellenos sanitarios en la salud no han sido concluyentes, se han documentado efectos potenciales en la salud de las poblaciones que residen en su área de influencia, especialmente en aquellas consideradas vulnerables, como es el caso de los adultos mayores. En particular, se ha reportado un aumento de la demanda de servicios de salud y en la presencia de síntomas respiratorios y exacerbaciones de procesos crónicos, como la enfermedad pulmonar obstructiva crónica y el asma, con un aumento en los síntomas percibidos. Además, la exposición a los contaminantes generados en los rellenos sanitarios se ha asociado a una reducción de los parámetros de la función pulmonar, como el volumen espiratorio forzado en un segundo $\left(\mathrm{VEF}_{1}\right)$ y el flujo espiratorio pico o máximo (5-10).

Los municipios de Cali, Yumbo y Jamundí producen unas 1.600 toneladas diarias de residuos sólidos que, entre 1967 y 2008, fueron dispuestos en el Botadero de Navarro. En las áreas vecinas al botadero ha ocurrido un proceso de urbanización acelerado y, por consiguiente, de exposición potencial de la población a los contaminantes emitidos a través del aire y de otras rutas de exposición, como agua y suelo.

Este estudio hace parte de una investigación desarrollada por el Grupo de Epidemiología y Salud Poblacional (sic.), en el 2005, para evaluar el impacto del Botadero de Navarro en la salud de los grupos de población residentes en su área de influencia. Esta investigación encontró un retraso del crecimiento físico infantil, incremento de la presencia de síntomas respiratorios en niños menores de cinco años y una mayor percepción del riesgo de enfermedades respiratorias en la población vecina al botadero (11-14). El Grupo llevó a cabo una evaluación con hallazgos similares en el relleno sanitario Doña Juana de Bogotá (resultados sin publicar).

Este artículo muestra los resultados de la evaluación del impacto del Botadero de Navarro sobre la salud respiratoria de los adultos mayores de 50 años residentes en el área de influencia. En particular, interesaba evaluar el impacto en la presencia y recurrencia de episodios agudos de diversa gravedad, en una población caracterizada por el bajo acceso a los servicios de salud.

\section{Materiales y métodos}

Esta investigación hizo parte de un estudio mayor en el que se evaluaba el impacto en la salud, en los costos en salud, en el impacto autopercibido en salud y en el ambiente físico y social en los grupos vulnerables de población (menores de 5 años y mayores de 50 años) que viven en el área de influencia del Botadero de Navarro (11-14).

En este artículo se reportan los resultados de un estudio prospectivo con seguimiento durante seis meses a una cohorte fija de adultos mayores de 50 años, residentes en el área de influencia del botadero, para evaluar la presentación mensual de síntomas respiratorios y alteraciones de la función pulmonar, en comparación con una cohorte fija de adultos en una zona no expuesta. Además, el estudio mayor incluyó la caracterización de los contaminantes en el aire (particulate matter, 10 $\left.\mu \mathrm{m}, \mathrm{PM}_{10}\right)$, dióxido de azufre $\left(\mathrm{SO}_{2}\right)$, metano $\left(\mathrm{CH}_{4}\right)$, xileno y benceno mediante controles en las dos zonas (11).

El presente estudio cumplió con lo estipulado en la declaración de Helsinki y por la Resolución 8430 del Ministerio de Salud, y fue avalado por el Comité de Ética de la Facultad de Salud de la Universidad del Valle.

\section{Área y población de estudio}

El estudio se hizo en una zona considerada como expuesta por su alta probabilidad de ser influenciada por las emisiones del botadero y en una zona distante seleccionada como no expuesta.

Se definió como grupo de expuestos a los sujetos residentes en varios barrios ubicados a $3 \mathrm{~km}$ o menos del perímetro del botadero, donde las condiciones meteorológicas (dirección predominante del viento) y topográficas (falta de barreras físicas) indicaban una alta probabilidad de exposición a los contaminantes emitidos desde el botadero, y en quienes la permanencia en el área de residencia fuera, al menos, de $80 \%$ durante el tiempo de seguimiento.

Como grupo no expuesto, se seleccionaron individuos residentes a más de $3 \mathrm{~km}$ del botadero, cuyas condiciones meteorológicas y topográficas indicaban baja probabilidad de exposición a los contaminantes, y con permanencia en su área de residencia, por lo menos, $80 \%$ del tiempo diario. Se procuró, además, que las otras características ambientales, topográficas y socioeconómicas (distribución urbana o rural, estratos 2, 3 y 4) de las zonas fueran similares 0 , en otras palabras, que la mayor diferencia entre ellas fuera su ubicación con relación al botadero. 
Por consiguiente, se seleccionaron como "expuestos", el corregimiento de Navarro y los barrios Morichal de Comfandi, Ciudad Córdoba y Caney, mientras que en la zona no expuesta se incluyeron el corregimiento del Hormiguero y los barrios Departamental, Cristóbal colón, León XIII y Conquistadores (figura 1).

El tamaño de la muestra se estimó asumiendo una proporción de síntomas respiratorios de $20 \%$ en la población adulta no expuesta (15-17), un nivel de significancia de 0,05 , un poder estadístico de $80 \%$, un efecto esperado (riesgo relativo) por la exposición igual o mayor de 1,5 y un total de cinco visitas planeadas para cada sujeto. El tamaño de la muestra finalmente estimado fue de 300 individuos mayores de 50 años para cada uno de los grupos expuesto y no expuesto.

\section{Diseño de muestreo}

Para la selección de la población de estudio, se hizo un muestreo por conglomerados en una etapa. Los conglomerados se constituyeron por las manzanas o agregados de viviendas. Según estimados del número de habitantes por vivienda por grupos de edad realizado por el DANE para cada comuna de Cali en el 2005, se calculó un promedio de 20 manzanas seleccionadas por barrio, para completar el tamaño de muestra de 300 adultos mayores de 50 años para cada zona de estudio. Para cada barrio se seleccionaron aleatoriamente las manzanas y se incluyeron los adultos mayores de 50 años de todas las viviendas que conformaron los conglomerados seleccionados y que cumplieron con los criterios descritos.

\section{Medición de los eventos}

Como "eventos en salud" se consideraron los síntomas respiratorios autorreportados, como rino-rrea, prurito nasal, estornudos, lagrimeo, disfonía, sibilancias, dificultad respiratoria, tos seca y tos húmeda o productiva en el día o en la noche, cuya incidencia fue medida mensualmente a partir de un cuestionario diseñado con base en el instrumento principal del ECRHS II (European Community Respiratory Health Survey II) $(18,19)$, que fue probado y ajustado a las condiciones locales antes de su uso.

Con una periodicidad mensual y simultáneamente a la aplicación del cuestionario, se midió el flujo

\section{Localización del Botadero de Navarro, Zona Expuesta y Zona Control Cali - Colom bia}

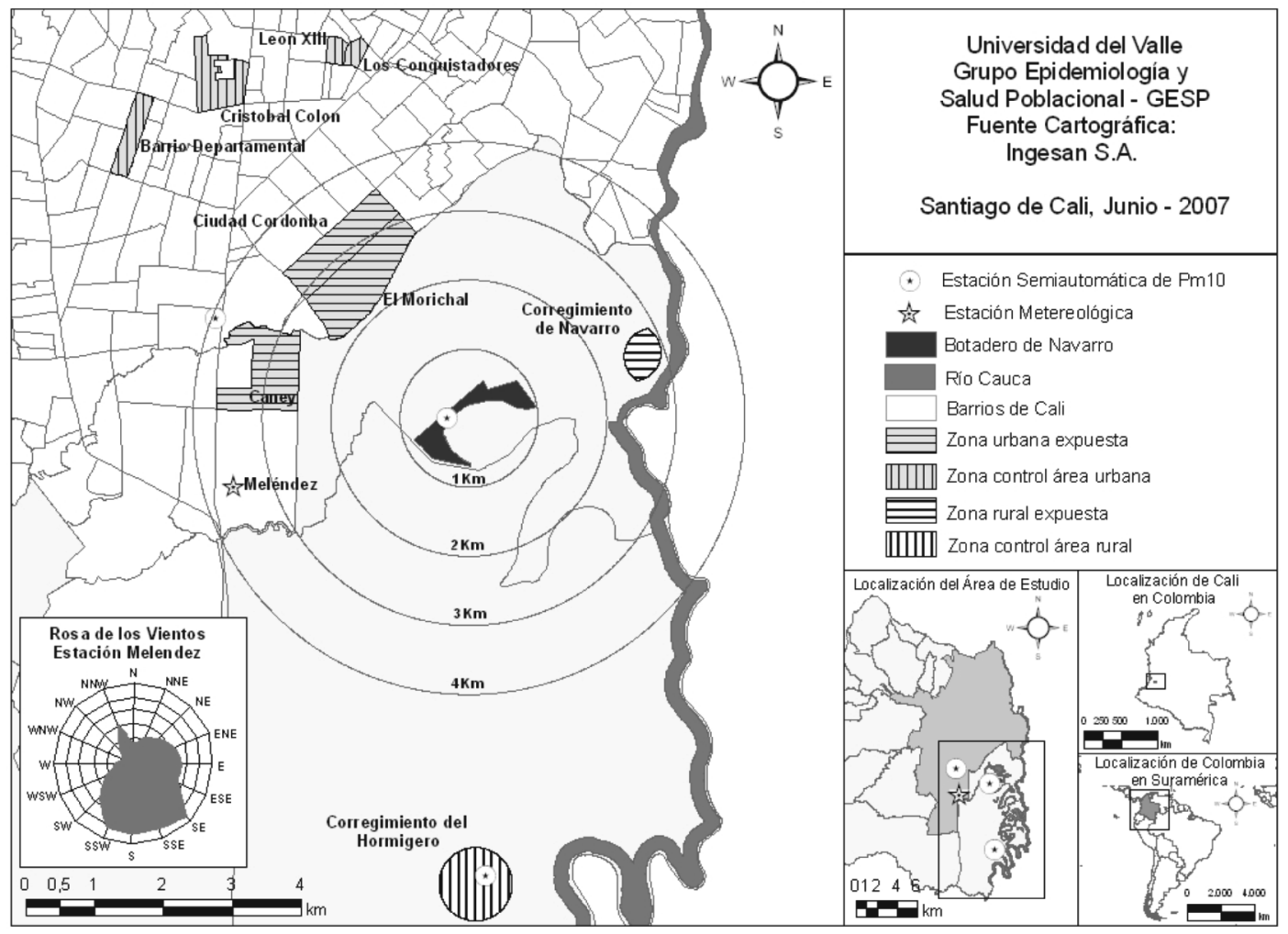

Figura 1. Localización de las zonas de estudio 
espiratorio pico. Ésta es una medida de la función pulmonar que corresponde a la máxima cantidad de aire que una persona puede movilizar durante una espiración forzada. Los valores inferiores a lo esperado, de acuerdo con factores como la edad, el sexo y la talla, indican una obstrucción de las vías aéreas de mediano y gran calibre $(20,21)$. Para su medición, el personal de campo fue capacitado y se usaron medidores portátiles de flujo espiratorio pico. Siguiendo las indicaciones para su uso clínico, y con el objetivo de asegurar una mayor validez, se realizó tres veces el procedimiento en cada persona y, para los análisis, se usó el mayor valor obtenido ajustado por la talla $(22,23)$.

Además, mediante una encuesta se determinaron otras variables que pueden influir en la presencia de síntomas respiratorios y en los valores del flujo espiratorio pico, tales como características sociodemográficas, antecedentes de exposición laboral, hábitos, antecedentes de enfermedades concomitantes(enfermedadescrónicasqueprodujeran malestares en el último mes y hospitalizaciones en los últimos 12 meses) y condiciones de la vivienda. Estas condiciones, que incluyeron los materiales de construcción de la casa, las fuentes de contaminación dentro de la vivienda y la presencia de moho y humedad, entre otros, se determinaron mediante la observación directa del interior de la vivienda por parte de las encuestadoras.

El personal de campo fue previamente capacitado y entrenado, con el objetivo de asegurar la estandarización del proceso de recolección de información. Antes de la aplicación de las encuestas, se hizo una prueba piloto en Cali a una población con características similares a las incluidas en el estudio y, de acuerdo con los resultados obtenidos en la prueba piloto, se hicieron los ajustes pertinentes al instrumento.

\section{Captación y seguimiento de la población de estudio}

Una vez seleccionada la población de estudio, se hizo una visita inicial para obtener el consentimiento informado y recolectar información correspondiente a la línea de base. Durante los seis meses siguientes, se realizó el seguimiento a la población seleccionada, expuesta y a la no expuesta, con visitas mensuales hasta el final del seguimiento.

\section{Análisis de los datos}

Se determinó la incidencia acumulada de síntomas respiratorios, de síntomas respiratorios moderados y graves, el promedio del flujo espiratorio pico, con sus respectivos intervalos de confianza del $95 \%$. La variable de síntomas respiratorios moderados y graves se construyó con base en la agrupación de los síntomas de acuerdo con sus características fisiopatológicas y su gravedad. De esta forma, los síntomas se consideraron moderados cuando en un solo individuo se presentaron síntomas broncoobstructivos o de bronquitis crónica; mientras que se consideraron graves cuando se presentaron simultáneamente síntomas broncoobstructivos, bronquíticos y de las vías aéreas superiores.

Para los análisis de asociación, dada la naturaleza repetitiva de las mediciones, se hicieron análisis de regresión con métodos para datos longitudinales (ecuaciones de estimación generalizadas, con uso de métodos de promedios de población y efectos aleatorios) que tienen en cuenta la correlación existente entre los datos por ser mediciones hechas en un mismo individuo (24). Dependiendo del tipo de variable respuesta, se utilizó un modelo logístico (variables categóricas como presencia de síntomas) o lineal (variables continuas como el flujo espiratorio pico).

Inicialmente, se estimaron las asociaciones univariadas entre la exposición principal, cada una de las variables y los eventos respiratorios. Para controlar por potenciales factores de confusión, se construyeron modelos de regresión múltiple en los que se incluyeron inicialmente las variables con coeficientes con una significancia menor de 0,25 en el análisis de univariado (25). Para la evaluación de modificación del efecto, se hicieron análisis estratificados o se incluyeron términos de interacción en los modelos de regresión múltiple. El término que tuviera una significancia menor de 0,05 fue incluido en el modelo final. Además, se examinó la presencia de "colinealidad" (colinearity) mediante los coeficientes de correlación de Spearman o de la estimación del factor de inflación de la varianza para variables categóricas y continuas, respectivamente. El modelo final se hizo guiado por algoritmos estadísticos conocidos como de stepwise backward (25) para la selección de variables, con una probabilidad de retiro mayor de 0,10 .

\section{Resultados}

Se incluyeron 672 individuos mayores de 50 años, 313 de ellos residentes en la zona de influencia y que cumplían con los criterios para clasificarlos como expuestos. El promedio de edad de los expuestos y en el grupo no expuesto fue de $65 \pm$ 8,8 y $62 \pm 9,3$ años, respectivamente. Las medianas 
Cuadro 1. Características de los adultos mayores de 50 años incluidos en la cohorte.

\begin{tabular}{|c|c|c|c|c|c|}
\hline \multirow[t]{2}{*}{ Variables } & \multicolumn{2}{|c|}{ Expuesto } & \multicolumn{2}{|c|}{ No expuesto } & \multirow[t]{2}{*}{$\mathbf{P}$} \\
\hline & $n=313$ & $\%$ & $n=359$ & $\%$ & \\
\hline \multicolumn{6}{|l|}{ Sociodemográficas } \\
\hline \multicolumn{6}{|l|}{ Sexo } \\
\hline Masculino & 103 & 32,9 & 128 & 35,6 & 0,454 \\
\hline Femenino & 210 & 67,1 & 231 & 64,4 & \\
\hline \multicolumn{6}{|l|}{ Edad (años) } \\
\hline 50 a 59 & & 134 & 42,8 & 7019,5 & \\
\hline 60 a 69 & & 102 & 32,6 & 15543,2 & 0,000 \\
\hline$\geq 70$ & & 77 & 24,6 & 13437,3 & \\
\hline \multicolumn{6}{|l|}{ Afiliación a seguridad social } \\
\hline No afiliado & 70 & 22,4 & 54 & 15,0 & 0,015 \\
\hline Afiliado & & 243 & 77,6 & 30585,0 & \\
\hline \multicolumn{6}{|l|}{ Régimen de afiliación } \\
\hline Contributivo & 169 & 69,5 & 163 & 53,4 & 0,000 \\
\hline Subsidiado & 74 & 30,5 & 142 & 46,6 & \\
\hline \multicolumn{6}{|l|}{ Escolaridad } \\
\hline Ninguna & & 33 & 10,5 & 4713,1 & \\
\hline Primaria & & 205 & 65,5 & 23264,6 & 0,491 \\
\hline Secundaria o más & 75 & 24,0 & 80 & 22,3 & \\
\hline \multicolumn{6}{|l|}{ Situación laboral } \\
\hline Empleados-independiente & 74 & 23,6 & 81 & 22,6 & \\
\hline Pensionado-incapacitado & 31 & 9,9 & 62 & 17,3 & 0,039 \\
\hline Ama de casa & 177 & 56,6 & 175 & 48,8 & \\
\hline Desempleado & 31 & 9,9 & 41 & 11,4 & \\
\hline \multicolumn{6}{|l|}{ De exposición laboral } \\
\hline \multicolumn{6}{|l|}{ Riesgo laboral entre empleados } \\
\hline Sin riesgo respiratorio & 62 & 83,8 & 58 & 71,6 & 0,024 \\
\hline Con riesgo respiratorio & 12 & 16,2 & 23 & 28,4 & \\
\hline \multicolumn{6}{|c|}{ Retiro de algún trabajo por síntomas respiratorios } \\
\hline No se retiró & 295 & 94,2 & 345 & 96,1 & 0,261 \\
\hline Se retiró & & 18 & 5,8 & 143,9 & \\
\hline \multicolumn{6}{|l|}{ Condiciones de la vivienda } \\
\hline \multicolumn{6}{|l|}{ Número de personas en la casa } \\
\hline 0 a 3 & & 88 & 28,1 & 13236,8 & \\
\hline 4 a 6 & & 145 & 46,3 & 10930,4 & 0,000 \\
\hline$\geq 7$ & & 80 & 25,6 & 11832,9 & \\
\hline \multicolumn{6}{|l|}{ Humedad } \\
\hline No & & 265 & 84,7 & 25772,2 & 0,000 \\
\hline Sí & & 48 & 15,3 & 9927,8 & \\
\hline \multicolumn{6}{|l|}{ Moho } \\
\hline No & & 303 & 96,8 & 32791,3 & 0,003 \\
\hline Sí & & 10 & 3,2 & 318,7 & \\
\hline \multicolumn{6}{|l|}{ Animales } \\
\hline No & & 136 & 43,4 & 14039,1 & 0,254 \\
\hline Sí & & 177 & 56,5 & 21860,1 & \\
\hline Fuente de cocina & & & & & \\
\hline Otra fuente sin riesgo respiratorio & 269 & 85,9 & 249 & 70,1 & 0,775 \\
\hline Carbón-leña, gasolina-gas & 44 & 14,1 & 106 & 29,9 & \\
\hline Material de las paredes & & & & & \\
\hline Ladrillo & & 284 & 90,7 & 31688,0 & 0,254 \\
\hline Otros materiales & 29 & 9,3 & 43 & 12,0 & \\
\hline Material del piso & & & & & \\
\hline Baldosa & & 244 & 78,0 & 22161,6 & 0,000 \\
\hline Ladrillo, cemento o tierra-arena & 69 & 22,0 & 138 & 38,4 & \\
\hline
\end{tabular}




\begin{tabular}{|c|c|c|c|c|c|}
\hline \multicolumn{6}{|c|}{$\begin{array}{l}\text { Hábitos y estilos de vida } \\
\text { Consumo de cigarrillo }\end{array}$} \\
\hline No fumador & 148 & 47,3 & 171 & 47,6 & \multirow{3}{*}{0,433} \\
\hline Ex fumador & 120 & 38,3 & 148 & 41,2 & \\
\hline Fumador actual & 45 & 14,4 & 40 & 11,2 & \\
\hline \multicolumn{6}{|c|}{ Edad (años) al inicio del consumo de cigarrillo } \\
\hline$>10$ & 151 & 91,5 & 168 & 89,4 & \multirow[t]{2}{*}{0,439} \\
\hline$\leq 10$ & 14 & 8,5 & 20 & 10,6 & \\
\hline \multicolumn{6}{|c|}{ Cercanía a cultivos de caña de azúcar (km) } \\
\hline$>1$ & 313 & 100 & 243 & 67,7 & \multirow[t]{2}{*}{0} \\
\hline$<1$ & 0 & 0 & 116 & 32,3 & \\
\hline
\end{tabular}

de tiempo de residencia en el barrio fueron de 4 y 30 años en el grupo expuesto y el no expuesto, respectivamente.

Durante el seguimiento se produjeron pérdidas similares en los grupos de comparación. Específicamente, desde la captación a la última visita de seguimiento, se produjeron $29,7 \%$ de pérdidas en la zona no expuesta y $32,3 \%$ en la zona expuesta. Las principales causas de las pérdidas fueron las mismas en los dos grupos en comparación: el rechazo a continuar participando en el estudio y el cambio de domicilio.

Los expuestos tendieron a ser más jóvenes que el grupo no expuesto: promedio de edad 63,1 [desviación estándar $(\mathrm{DE})=9,3)]$ Vs. 66,7 (DE=8,8), mientras la distribución por sexo fue similar en ambos grupos. Además, los expuestos tenían mejores condiciones socioeconómicas, como se ve reflejado en una mayor afiliación al régimen contributivo, mayor proporción de personas laboralmente activas y mejores condiciones de la vivienda (cuadro 1). En comparación con el grupo expuesto, el no expuesto tenía un mayor número de personas por familia (7 o más personas), mayor proporción de viviendas con humedad, con moho y con animales en la vivienda, mayor tendencia a cocinar con carbón, leña, gasolina o gas propano y a desempeñar funciones consideradas de riesgo para el sistema respiratorio (construcción, ebanistería, areneros, pintores y zapateros). Los expuestos y los no expuestos fueron similares en cuanto a factores como el consumo de cigarrillo y la presencia de enfermedades concomitantes o discapacidad.

A la captación, se encontró una mayor prevalencia de síntomas respiratorios en todos los grados (leve, moderado y grave) en el grupo expuesto (26,5\%, $28,1 \%$ y $25,9 \%$, respectivamente), que en el grupo no expuesto (22 \%, 22,6 \% y $20,6 \%$, respectivamente).

Luego de ajustar por otras variables, la exposición al botadero se encontró estadísticamente asociada como un factor de riesgo independiente para la incidencia de síntomas respiratorios, con una probabilidad $25 \%$ mayor de presencia de síntomas en comparación con el grupo no expuesto $(\mathrm{OR}=1,25)$. Se encontró también un gradiente de asociación positivo entre la presencia de síntomas respiratorios y el aumento de la edad. Las personas sin afiliación a la seguridad social tuvieron $47 \%$ mayor oportunidad de presentar síntomas respiratorios, en comparación con las afiliadas a los regímenes subsidiado o contributivo (cuadro 2).

El no tener ningún nivel de escolaridad, la exposición ocupacional previa y la presencia de humedad en la vivienda, también se asociaron con la incidencia de estos síntomas (cuadro 2). Las personas que iniciaron el consumo de cigarrillo a edad temprana (antes de los 10 años) tenían más del doble de la oportunidad de síntomas respiratorios en comparación con los que nunca habían fumado (cuadro 2). Durante el seguimiento se determinó, además, la existencia de una mayor repetición de cuadros respiratorios con múltiples síntomas por individuo. Específicamente, los resultados muestran una agregación significativa en la presencia de múltiples síntomas respiratorios por individuo (coeficiente de correlación en la clase de 0,$\left.28 ; I C_{95 \%} ; 0,23-0,33 ; p=0,000\right)$.

Después de ajustar por las variables de interés, se encontró asociación entre la exposición al botadero y la presencia de síntomas respiratorios moderados. Así, los expuestos al botadero tuvieron $28 \%$ mayor oportunidad de estos síntomas, en comparación con el grupo no expuesto (cuadro 3). La presencia de síntomas respiratorios moderados también se asoció a la residencia cercana a los cultivos de caña de azúcar. Los sujetos residentes a menos de un kilómetro de los cultivos de caña de azúcar, tenían $27 \%$ mayor oportunidad de estos síntomas en comparación con los que residían a mayor distancia (cuadro 3). También, se encontró un mayor riesgo de síntomas moderados con una 
edad mayor, con la presencia de enfermedades concomitantes (como diabetes, hipertensión arterial, dolor corporal, artritis, entre otras), con la falta de afiliación a la seguridad social y con la presencia de animales en la vivienda (cuadro 3).

La presencia de síntomas respiratorios moderados fue $82 \%$ más probable entre los sujetos con presencia de humedad en la casa, en comparación con aquellos sin humedad en la vivienda. Además, los individuos con inicio temprano del consumo de cigarrillo (habían empezado a fumar antes de los 10 años de edad) tenían $78 \%$ mayor oportunidad de síntomas moderados, en comparación con los que nunca habían fumado (cuadro 3).

Aunque se encontró una tendencia a mayor riesgo de síntomas respiratorios graves en los individuos expuestos, este hallazgo no fue estadísticamente significativo después de ajustar por variables sociodemográficas, condiciones de la vivienda y exposición ocupacional (OR=1,16; $\mathrm{IC}_{95 \%} ; 0,89$;1,51) (cuadro 3). De manera similar con los hallazgos para síntomas respiratorios moderados, se encontró mayor oportunidad de síntomas respiratorios graves en los sujetos con una mayor edad, sin ninguna afiliación a la seguridad social en salud, sin ningún nivel de escolaridad o que refirieron haberse retirado de algún trabajo por presentar síntomas respiratorios (estas dos últimas variables no se presentan en la tabla) (cuadro 3).

En cuanto a la función pulmonar, la exposición se asoció con una reducción del flujo espiratorio pico, después de ajustar por edad, sexo, talla, hábitos, exposición ocupacional, porcentaje de la vida con residencia en la zona y cercanía a cultivos de

Cuadro 2. Factores asociados a la incidencia de síntomas respiratorios (modelo de estimación de ecuaciones generalizadas de regresión logística)

\begin{tabular}{|c|c|c|}
\hline Variable & $\begin{array}{c}\text { OR }\left(\text { IC }_{95 \%}\right) \\
\text { crudo }\end{array}$ & $\begin{array}{l}\text { OR }\left(\text { IC }_{95 \%}\right) \\
\text { ajustado }\end{array}$ \\
\hline \multicolumn{3}{|l|}{ Botadero de Navarro } \\
\hline No expuesto & 1 & 1 \\
\hline Expuesto & $0,99(0,81-1,20)$ & $1,25(1,01-1,56)^{\star *}$ \\
\hline \multicolumn{3}{|c|}{ Cercanía a cultivos de caña de azúcar (km) } \\
\hline$>1$ & 1 & 1 \\
\hline$<1$ & $1,41(1,09-1,82)$ & $1,23(0,91-1,65)$ \\
\hline \multicolumn{3}{|l|}{ Edad (años) } \\
\hline 50 a 59 & 1 & 1 \\
\hline 60 a 69 & $1,46(1,16-1,85)$ & $1,43(1,12-1,84)^{\star \star *}$ \\
\hline$\geq 70$ & $2,07(1,61-2,66)$ & $1,87(1,43-2,45)^{\star * *}$ \\
\hline \multicolumn{3}{|c|}{ Enfermedad concomitante } \\
\hline No & 1 & 1 \\
\hline Sí & $1,26(1,02-1,54)$ & $1,16(0,94-1,43)$ \\
\hline \multicolumn{3}{|l|}{ Afiliación } \\
\hline Afiliado & 1 & 1 \\
\hline No afiliado & $1,32(1,02-1,71)$ & $1,47(1,13-1,91)^{\star * *}$ \\
\hline \multicolumn{3}{|l|}{ Escolaridad } \\
\hline Secundaria o más & 1 & 1 \\
\hline Primaria & $1,23(0,97-1,56)$ & $1,04(0,82-1,32)$ \\
\hline Ninguna & $2,13(1,48-3,07)$ & $1,68(1,16-2,43)^{\star * *}$ \\
\hline \multicolumn{3}{|c|}{ Retiro de un trabajo por síntomas respiratorios } \\
\hline No & 1 & 1 \\
\hline Sí & $2,06(1,25-3,37)$ & $1,83(1,12-2,99)^{\star *}$ \\
\hline \multicolumn{3}{|l|}{ Humedad } \\
\hline No & 1 & 1 \\
\hline Sí & $1,66(1,31-2,12)$ & $1,71(1,34-2,18)^{\star * *}$ \\
\hline \multicolumn{3}{|c|}{ Edad (años) al inicio del cigarrillo } \\
\hline Nunca ha fumado & 1 & 1 \\
\hline$>10$ & $1,09(0,88-1,35)$ & $1,00(0,82-1,23)$ \\
\hline$\leq 10$ & $1,06(0,79-1,45)$ & $2,10(1,27-3,48)^{\star * *}$ \\
\hline
\end{tabular}

${ }^{*}: 0,05<p>0,10 ;{ }^{* *}: 0,01<p<0,05 ;{ }^{* * *}: p<0,01$ 
Cuadro 3. Factores asociados a la incidencia de síntomas respiratorios moderados y síntomas respiratorios graves (modelo de estimación de ecuaciones generalizadas de regresión logística)

\begin{tabular}{|c|c|c|}
\hline Variable & $\begin{array}{c}\text { Síntomas respiratorios } \\
\text { moderados } \\
\text { OR§ }\left(\mathrm{IC}_{95 \%}\right) \\
\text { ajustado }\end{array}$ & $\begin{array}{c}\text { Síntomas respiratorios } \\
\text { graves } \\
\text { OR† (IC } \\
\text { Ajustado }\end{array}$ \\
\hline $\begin{array}{l}\text { Botadero de Navarro } \\
\text { No expuesto } \\
\text { Expuesto }\end{array}$ & $\begin{array}{c}1 \\
1,28(0,98-1,66)^{*}\end{array}$ & $\begin{array}{c}\mathbf{1} \\
1,16(0,89-1,51)\end{array}$ \\
\hline $\begin{array}{l}\text { Cercanía a cultivos de } \\
>1 \\
<1\end{array}$ & $\begin{array}{c}1 \\
1,27(1,06-2,05)^{\star *}\end{array}$ & $\begin{array}{c}1 \\
1,28(0,91-1,80)\end{array}$ \\
\hline $\begin{array}{l}\text { Edad (años) } \\
\quad 50 \text { a } 59 \\
60 \text { a } 69 \\
\geq 70\end{array}$ & $\begin{array}{c}1 \\
1,34(1,01-2,05)^{* *} \\
1,44(1,06-1,97)^{\star *}\end{array}$ & $\begin{array}{c}1 \\
1,17(0,86-1,57) \\
1,56(1,14-2,14)^{\star * \star}\end{array}$ \\
\hline $\begin{array}{l}\text { Enfermedades conco } \\
\text { No } \\
\text { Sí }\end{array}$ & $\begin{array}{c}1 \\
1,27(1,00-1,63)^{*}\end{array}$ & $\begin{array}{c}1 \\
1,20(0,94-1,54)\end{array}$ \\
\hline $\begin{array}{l}\text { Afiliación } \\
\text { Afiliado } \\
\text { No afiliado }\end{array}$ & $\begin{array}{c}1 \\
1,58(1,18-2,12)^{\star \star *}\end{array}$ & $\begin{array}{c}1 \\
1,39(1,03-1,89)^{\star *}\end{array}$ \\
\hline $\begin{array}{l}\text { Humedad } \\
\text { No } \\
\text { Sí }\end{array}$ & $\begin{array}{c}1 \\
1,82(1,40-2,38)^{\star * *}\end{array}$ & $\begin{array}{c}1 \\
2,12(1,61-2,79)^{* * *}\end{array}$ \\
\hline $\begin{array}{l}\text { Edad (años) al inicio c } \\
\text { Nunca ha fumado } \\
>10 \\
\leq 0\end{array}$ & $\begin{array}{c}1 \\
0,96(0,76-1,22) \\
1,78(1,08-2,94)^{\star *}\end{array}$ & $\begin{array}{c}1 \\
1,75(1,36-2,25)^{\star * *} \\
2,83(1,58-5,09)^{\star * *}\end{array}$ \\
\hline
\end{tabular}

${ }^{*}: 0,05<p>0,10 ;{ }^{* *}: 0,01<p<0,05 ;{ }^{* * *}: p<0,01$

$\S$ : ajustado por presencia de animales en la casa; $\uparrow$ : ajustado por sexo, escolaridad, retiro de algún trabajo por síntomas respiratorios y número de personas en la casa.

caña. Específicamente, durante el seguimiento, los expuestos tuvieron en promedio 15,19 litros por minuto menos de flujo espiratorio pico, en comparación con el grupo no expuesto (cuadro 4).

Respecto a la edad, se encontró una reducción en el flujo espiratorio pico de $-3,55$ litros por minuto, por cada año de edad $\left(\mathrm{IC}_{95 \%} ;-4,22\right.$ a $\left.-2,89\right)$. Los hombres tenían, en promedio, 85,17 litros por minuto más de flujo espiratorio pico que las mujeres $\left(\mathrm{IC}_{95 \%} ; 69,6-100,8\right)$ (cuadro 4).

El tiempo de residencia en el barrio se asoció a un menor valor de flujo espiratorio pico. Las personas que llevaban el $25 \%$ o más de su vida en el barrio tenían en promedio 28,4 litros por minuto menos de flujo espiratorio pico, en comparación con los sujetos que llevaban menos de este tiempo viviendo en la zona (cuadro 4). Además, se asociaron a menores valores promedios de flujo espiratorio pico, la presencia de síntomas respiratorios moderados y graves, y haberse retirado de algún trabajo por síntomas respiratorios. Aunque hubo una tendencia a menor valor de flujo espiratorio pico en los sujetos que habían empezado a fumar antes de los 10 años de edad, en comparación con los que nunca habían fumado (-22,15 litros por minuto), esta diferencia no fue estadísticamente significativa $(p=0,120)$ (cuadro 4$)$.

En la evaluación de modificación del efecto, se encontró una mayor presencia de síntomas respiratorios graves por la exposición, en los sujetos sin afiliación a la seguridad social en salud. Específicamente, entre los no afiliados, la exposición se asoció a un aumento de 6,6 veces en la oportunidad de síntomas graves en comparación con los sujetos en la zona no expuesta. Por el contrario, entre los afiliados, la exposición aumentó sólo 1,3 veces la oportunidad de síntomas graves ( $p$ interacción $=0,005$ ) (cuadro 5).

Además, se encontró un mayor efecto del botadero entre los sujetos de la zona expuesta que llevaban mayor tiempo de residencia en el barrio. Los expuestos que llevaban más de $25 \%$ de su vida 
Cuadro 4. Factores asociados a la diferencia promedio del flujo espiratorio pico entre expuestos y no expuestos al Botadero de Navarro (modelo de estimación de ecuaciones generalizadas, de regresión lineal)

\begin{tabular}{|c|c|c|}
\hline Variable & $\begin{array}{c}\text { Diferencia promedio del } \\
\text { flujo espiratorio pico } \\
\left(\mathrm{IC}_{95 \%}\right) \text { cruda }\end{array}$ & $\begin{array}{c}\text { Diferencia promedio del } \\
\text { flujo espiratorio pico } \\
\left(\mathrm{IC}_{95 \%}\right) \text { ajustada§ }\end{array}$ \\
\hline \multicolumn{3}{|l|}{ Botadero de Navarro } \\
\hline No expuesto & Referencia & Referencia \\
\hline Expuesto & $10,46(-4,53-25,45)$ & $-15,19(-30,16-0,22)^{\star \star}$ \\
\hline \multicolumn{3}{|l|}{ Proporción de la vida de residencia en el barrio (\%) } \\
\hline$<25$ & Referencia & Referencia \\
\hline$\geq 25$ & $-28,32(-48,09$ a $-8,56)$ & $-28,97(-48,02 \text { a }-9,91)^{\star * *}$ \\
\hline \multicolumn{3}{|l|}{ Cercanía a cultivos de caña de azúcar (km) } \\
\hline$>1$ & Referencia & Referencia \\
\hline$<1$ & $-14,29(-33,93$ a $-5,34)$ & $-6,42(-23,54 a-10,71)$ \\
\hline Edad en años & $-3,66(-4,42 \mathrm{a}-2,92)$ & $-3,55(-4,22 \text { a }-2,89)^{\star \star *}$ \\
\hline \multicolumn{3}{|l|}{ Sexo } \\
\hline Femenino & Referencia & Referencia \\
\hline Masculino & $98,59(84,39-112,78)$ & $85,17(69,57-100,78)^{\star * *}$ \\
\hline \multicolumn{3}{|l|}{ Síntomas respiratorios } \\
\hline Ninguno o síntomas de vías aéreas superiores & Referencia & Referencia \\
\hline Síntomas moderados & $-16,98(-24,04$ a $-9,92)$ & $-12,17(-17,64 a-6,69)^{* * *}$ \\
\hline Síntomas graves & $-27,67(-36,26-19,08)$ & $-21,03(-27,69 ;-14,36)^{\star * *}$ \\
\hline \multicolumn{3}{|l|}{ Retiro de algún trabajo por síntomas respiratorios } \\
\hline No & Referencia & Referencia \\
\hline Sí & $-68,52(-102,82$ a $-34,24)$ & $-51,16(-78,05 a-4,27)^{\star * *}$ \\
\hline \multicolumn{3}{|l|}{ Edad (años) al inicio del cigarrillo } \\
\hline Nunca ha fumado & Referencia & Referencia \\
\hline$>10$ & $29,91(14,66-45,16)$ & $1,17(-11,27-13,61)$ \\
\hline$\leq 10$ & $12,06(-23,19-47,31)$ & $-22,15(-50,08-5,77)$ \\
\hline
\end{tabular}

${ }^{*}: 0,05<p>0,10 ;{ }^{* *}: 0,01<p<0,05 ;{ }^{* * *}: p<0,01$

$\S$ : ajustado por talla

Cuadro 5. Modificación del efecto de la afiliación a seguridad social en salud, sobre la presencia de síntomas y síndromes respiratorios, y sobre el tiempo de residencia en la zona, según el flujo espiratorio pico

\begin{tabular}{|c|c|c|c|c|}
\hline \multirow[b]{2}{*}{ Evento } & \multicolumn{3}{|c|}{ Síntomas respiratorios } & \multirow[b]{2}{*}{$\mathbf{p}$} \\
\hline & OR ajustado & $\begin{array}{c}\text { OR ajustado } \\
\text { entre los afiliados }\end{array}$ & $\begin{array}{c}\text { OR ajustado } \\
\text { entre los no afiliados }\end{array}$ & \\
\hline Uno o más síntomas respiratorios & 1,65 & 1,36 & 4,13 & 0,106 \\
\hline Síndromes moderados & 1,90 & 1,93 & 1,67 & 0,945 \\
\hline Síndromes graves & 1,70 & 1,27 & 6,63 & 0,005 \\
\hline \multicolumn{5}{|l|}{ Función pulmonar } \\
\hline Evento & FEP ajustado & $\begin{array}{l}\text { FEP ajustado } \\
<25 \%^{*}\end{array}$ & $\begin{array}{l}\text { FEP ajustado } \\
>25 \%^{\star \star}\end{array}$ & $\mathbf{p}$ \\
\hline FEP & $-17,85$ & $-3,63$ & $-43,44$ & 0,066 \\
\hline
\end{tabular}

FEP: flujo espiratorio pico; *: FEP ajustado entre sujetos con menos del $25 \%$ de su vida en la zona; **: FEP ajustado entre sujetos con $25 \%$ o más de su vida en la zona

residiendo en la zona, tenían 43,4 litros por minuto menos de flujo espiratorio pico, en comparación con los expuestos que habían vivido en la zona menos de $25 \%$ de su vida ( $p$ interacción $=0,06$ ) (cuadro 5).

\section{Discusión}

En esta cohorte de adultos mayores de 50 años residentes en la zona expuesta, se encontró una mayor frecuencia de síntomas respiratorios generales y síntomas respiratorios moderados, en comparación con los residentes en un área no expuesta. Se encontró, también, una tendencia a mayor presencia de síntomas respiratorios graves en los expuestos, pero esta diferencia no fue estadísticamente significativa. Además, en este estudio se evaluó el cambio en el flujo espiratorio pico con el tiempo y se identificó la presencia de 
enfermedad respiratoria y otras covariables que pudieran explicar parcial o totalmente los cambios en la función pulmonar.

En particular, se encontró que, después de ajustar por las variables de importancia, los individuos expuestos presentaron en promedio un menor valor en el flujo espiratorio pico que los individuos de la zona no expuesta. Esta asociación entre la presencia de síntomas respiratorios y la exposición al botadero, concuerda con lo descrito en otros estudios, sobre los efectos en la salud producidos por el tipo de contaminantes generados en los sitios de disposición final de residuos sólidos (26-28).

La presencia de síntomas respiratorios también se encontró asociada a otros factores descritos en la literatura científica, como factores de riesgo para la presenciadeenfermedadrespiratoriaenlapoblación adulta mayor, como son la edad, el sexo $(29,30)$, el consumo de cigarrillo $(31,32)$, la humedad en la vivienda (33-36), la condición socioeconómica (37$40)$, la afiliación a la seguridad social, la presencia de enfermedades concomitantes y otras fuentes de contaminación atmosférica como la ocupacional $(41,42)$ y la cercanía a cultivos de caña de azúcar, que son quemados para su cosecha (43).

En cuanto al flujo espiratorio pico, se han encontrado datos similares en otros estudios en población adulta, que muestran una disminución en la función pulmonar asociada con la exposición a largo plazo a partículas en el ambiente (total solid oarticles, TSP, y $\mathrm{PM}_{10}$ ) y a gases como $\mathrm{NO}_{2}$ y $\mathrm{SO}_{2}$. Estos estudios han reportado, además, que las alteraciones en el flujo espiratorio pico han demostrado ser indicadores sensibles de los efectos agudos de contaminantes como el $\mathrm{SO}_{2}$, en pacientes con enfermedad pulmonar obstructiva crónica $(23,44)$. En este estudio, la reducción del flujo espiratorio pico asociada a la exposición fue independiente de la presencia de síntomas respiratorios. Esto concuerda con los resultados encontrados por Zemp, et al., que reportaron reducciones del flujo espiratorio pico, tanto en sujetos expuestos a contaminantes ambientales con síntomas respiratorios como en sujetos libres de ellos (6-8). Además, los menores valores de flujo espiratorio pico se asociaban a la condición socioeconómica (45), a un mayor tiempo de residencia en el barrio y a la exposición ocupacional previa (46-48).

La fortaleza de la asociación causal en epidemiología ambiental está determinada, en parte, por la calidad de la medición de la exposición. En nuestro estudio, la determinación de la exposición fue ecológica, ya que se identificó un grupo de individuos con alta probabilidad de estar expuestos a las emisiones del botadero y se comparó con otro no expuesto a esas emisiones.

La comparación hecha por Gómez, et al. (11), entre la concentración de contaminantes atmosféricos en el área de influencia, en comparación con la zona no expuesta del estudio, demostró la presencia de material en partículas, metano y benceno en mayores concentraciones en los barrios seleccionados como "expuestos", cuyo origen fueron las emisiones. Ninguna otra fuente de contaminación industrial o de tráfico vehicular importante podría explicar las diferencias observadas entre las dos zonas de estudio. Además, el hecho de que se haya asegurado que los individuos incluidos habían permanecido en la zona de estudio correspondiente durante la mayor parte del tiempo (más de $80 \%$ del día), fortalece esta caracterización de la exposición y descarta la influencia de la movilidad de los individuos como un posible sesgo.

En este estudio se encontraron diferencias en el tiempo de residencia en el barrio entre expuestos y no expuestos, con 4 y 30 años, respectivamente. Estas diferencias pueden deberse a varias circunstancias. En primer lugar, los barrios que conforman la zona expuesta al Botadero de Navarro fueron construidos hace menos tiempo que los barrios de la zona no expuesta; además, esos barrios corresponden a la zona de expansión del municipio. En segundo lugar, es posible que una mayor percepción del riesgo para la salud de los sujetos de la zona expuesta o la aparición de síntomas de enfermedad, hayan motivado a la población a migrar rápidamente a otros barrios de la ciudad.

Por consiguiente, esto pudo haber generado una de las potenciales debilidades del estudio, como fue el número de pérdidas en los grupos "expuesto" y "no expuesto", que fueron de 32 y $29 \%$ al final del seguimiento, respectivamente, lo que podría ser fuente posible de sesgos. Sin embargo, es probable que en este estudio el efecto de dichas pérdidas fuera mínimo o estuviera a favor de las conclusiones descritas, es decir que se pudo haber generado una subestimación en las asociaciones encontradas. Lo anterior puede sustentarse en parte en que el volumen de las pérdidas en ambos grupos fue muy similar, lo que sugiere que no están asociadas a la exposición de interés.

Además, se pudo comprobar que los individuos más enfermos fueron quienes con mayor frecuencia 
dejaron de participar en el estudio. Este tipo de pérdidas ocurrió en mayor proporción en la zona expuesta que en la zona no expuesta, pues la incidencia de síntomas respiratorios generales y de síntomas respiratorios moderados y graves, disminuyó más en esta zona que en la no expuesta: para síntomas respiratorios, de 49,5 a $38 \%$ entre la primera y la última visita en la zona expuesta, y de 53 a $45 \%$ en la zona control; para síntomas moderados, de 20 a $14 \%$ en la zona expuesta y de 23 a $20 \%$ en la zona control; y para síntomas graves, de 12,5 a $8,9 \%$ en la zona expuesta y de 11 a $13,3 \%$ en la zona control. Por lo tanto, este sesgo puede estar a favor de las conclusiones del estudio, debido a que hipotéticamente podría haber generado una subestimación de las asociaciones encontradas y, por tanto, todas las asociaciones positivas podrían ser aún de mayor magnitud.

Otra debilidad del estudio podría ser la tendencia a un exceso de reportes de los eventos adversos entre la población expuesta. Se ha descrito que los individuos que viven cerca de los rellenos sanitarios tienden a reportar con mayor frecuencia la presencia de síntomas respiratorios y otros de tipo inespecífico (2). Sin embargo, este estudio incluyó mediciones de parámetros biológicos, como el flujo espiratorio pico, en el que se encontraron diferencias estadísticamente significativas entre los grupos de comparación, que no estarían sujetas a ese tipo de sesgos. Además, está a favor de la validez de las conclusiones del estudio, el que los hallazgos concuerdan con lo referido en la literatura científica sobre otras exposiciones o covariables evaluadas, por ejemplo, edad, consumo de cigarrillo, nivel socioeconómico, humedad en la vivienda y exposición ocupacional).

Entre las fortalezas del estudio se encuentran, en primer lugar, que el seguimiento prospectivo permitió identificar, con menor posibilidad de sesgos, los cambios en la presentación de los eventos y su posible deterioro durante las visitas que se llevaron a cabo en los seis meses del estudio. Lo anterior confiere mayor validez a la determinación de la incidencia de los eventos y permitió la medición de los cambios en la exposición por movilidad de los sujetos incluidos. Además, el seguimiento y la medición repetida de los efectos en la salud respiratoria, permitieron incrementar el poder estadístico del estudio.

En conclusión, este estudio presenta datos en favor de que, independientemente de los potenciales factores de confusión, la exposición afecta la salud respiratoria de los sujetos mayores de 50 años expuestos, con una mayor presencia y cronicidad de los síntomas respiratorios, en particular, de los síntomas respiratorios clasificados como moderados, y con una reducción del flujo espiratorio pico. La información encontrada es útil para los que toman decisiones, pues sugieren que la exposición se asocia a efectos específicos en la salud respiratoria de la población adulta mayo; en particular, para el diseño de estrategias para el fortalecimiento de las medidas técnicas orientadas a establecer y controlar las emisiones contaminantes, y al diseño de sistemas de vigilancia de los efectos en la salud de la población expuesta más vulnerable.

\section{Agradecimientos}

Especiales agradecimientos a las personas residentes en la zonas de estudio y a quienes aceptaron participar en la investigación. Al personal de trabajo de campo, a Hoover León, encargado de la administración de los datos, y a Nathalie Abrahams, por la administración del proyecto.

\section{Conflicto de intereses}

Los autores declaramos que no se presentó ningún conflicto de intereses para la realización de esta investigación.

\section{Financiación}

Esta investigación fue financiada por Colciencias (contrato 414-2004) y la Universidad del Valle.

\section{Referencias}

1. Johannessen L, Boyer G. Observations of solid waste landfills in developing countries: Africa, Asia, and Latin America. The International Bank for Reconstruction and Development. Washington D.C.: The World Bank; 1999.

2. Vrijheid M. Health effects of residence near hazardous waste landfill sites: A review of epidemiologic literature. Environ Health Perspect. 2000;108:101-12.

3. Fielder HMP, PoonKing CM, Palmer CR, Moss N, Coleman G. Assessment of impact on health of residents living near the NantyGwyddon landfill site: Retrospective analysis. BMJ. 2000;320:19-23.

4. Cristina L, Sisinno S. Non-inert industrial solid waste disposal in landfill dumps: Evaluation of toxicity and implications for the environment and human health. Cad Saúde Pública. 2003;19:369-74.

5. Rushton L. Health hazards and waste management. $\mathrm{Br}$ Med Bull. 2003;68:183-97.

6. Zemp E, Elsasser S, Schindler C, Künzli N, Perruchoud AP, Domenighetti G, et al. Long term ambient air pollution and respiratory symptoms in adults (SAPALDIA study). The SAPALDIA Team. Am J Respir Crit Care Med. 1999;159:1257-66. 
7. Neuberger M, Schimek M, Horak JR, Moshammerb H, Kundib M, Frischer T, et al. Acute effects of particulate matter on respiratory diseases, symptoms and functions: Epidemiological results of the Austrian Project on Health Effects of Particulate Matter (AUPHEP). Atmos Environ. 2004;38:3971-81.

8. Briggs D. Environmental pollution and the global burden of disease. Br Med Bull. 2003;68:1-24.

9. Katsouyanni K. Ambient air pollution and health. Br Med Bull. 2003;68:143-56.

10. Sunyer J. Urban air pollution and chronic obstructive pulmonary disease: A review. Eur Respir J. 2001;17:102433.

11. Gómez R, Filigrana P, Méndez F. Descripción de la calidad del aire en el área de influencia del Botadero de Navarro, Cali, Colombia. Colombia Médica. 2008;39:245-52.

12. Mosquera J, Gómez O, Méndez F. Percepción del impacto del vertedero final de basuras en la salud y en el ambiente físico y social en Cali. Rev Salud Pública. 2009;11:549-58.

13. Girón S, Mateus J, Méndez F. Impacto de un botadero a cielo abierto en el desarrollo de síntomas respiratorios y en costos familiares de atención en salud de niños entre 1 a 5 años en Cali, Colombia. Biomédica. 2009;29:392-402.

14. Ocampo C, Pradilla A, Méndez F. Impacto de un depósito de residuos sólidos en el crecimiento infantil. Colombia Médica. 2008;39:245-54.

15. Frank PI, Wicks PD, Hazell ML, Linehan MF, Hirsch S, Hannaford PC, et al. Temporal change in the prevalence of respiratory symptoms and obstructive airways disease 1993-2001. Br J Gen Pract. 2005;55:596-602.

16. Linehan MF, Hazell ML, Frank TL, Frank PI. Prevalence of respiratory symptoms in under 5s: 1993 to 2001. Arch Dis Child. 2005;90:516-9.

17. Tabak C, Smit HA. Bronchial symptoms and obstruction: Recent prevalence and short-term trends (1993-1997) in adults in the Netherlands. Ned Tijdschr Geneeskd. 2001;145:2429-34

18. Burney P, Luczynsca J, Chinn S, Jarvis D. The European community respiratory health survey. Eur Respir J. 1994;7:954-60.

19. Bellia V, Pistelli F, Giannini D, Scichilone N, Catalano F, Spatafora M, et al. Questionnaires, spirometry and FEP monitoring in epidemiological studies on elderly respiratory patients. Eur Respir J. 2003;21 (Suppl.40):21-7.

20. Gregg A, Nunn AJ. Peak expiratory flow in normal subjects. Br Med J. 1973;3:382-84.

21. Stocks J, Quanjer PH. Reference values for residual volume, functional residual capacity and total lung capacity. ATS workshop on lung volume measurements. Official statement of the European Respiratory Society. Eur Respir J. 1995;8:492-506

22. Ruffin R. Peak expiratory flow (FEP) monitoring. Thorax. 2004;59:913-4

23. Douma WR, Mark W, Folgering H, Kort E, Koëter GH, Postma DS. Mini-wright peak flow meters are reliable after 5 years use. Eur Respir J. 1997;10:457-9.
24. Zeger SL, Liang KY. Longitudinal data analysis for discrete and continues outcomes. Biometrics. 1986;42:121-30.

25. Hosmer DW, Lemeshow S. Applied logistic regression. New York: John Wiley \& Sons; 1989.

26. Wong T, Wun Y, Yu T, Tam W, Wong C, Wong A. Air pollution and general practice consultations for respiratory illnesses. J Epidemiol Community Health. 2002;56:949-50.

27. Hernández-Cadena L, Téllez-Rojo M, Sanín-Aguirre L, Lacasaña-Navarro M, Campos A, Romieu I. Relación entre consultas a urgencias por enfermedad respiratoria y contaminación atmosférica en Ciudad Juárez, Chihuahua. Salud Pública Mex. 2000:42:288-97.

28. Hegde U, Chang TC, Yang SS. Methane and carbon dioxide emission from Shan-Chu-Ku landfill site in northern Taiwan. Chemosphere. 2003;52:1275-85.

29. Eagan TM, Bakke PS, Eide GE, Gulsvik A. Incidence of asthma and respiratory symptoms by sex, age and smoking in a community study. Eur Respir J. 2002;19:599-605.

30. Ronmark E, Lundback B, Jönsson E, Jonsson A-C, Lindström M, Sandstöm T, et al. Incidence of asthma in adults-report from the Obstructive Lung Disease in Northern Sweden Study. Allergy. 1997;52:1071-8.

31. Vineis P, Airoldi L, Veglia F, Olgiati L, Pastorelli R, Autrup $\mathbf{H}$, et al. Environmental tobacco smoke and risk of respiratory cancer and chronic obstructive pulmonary disease in former smokers and never smokers in the EPIC prospective study. BMJ. 2005;330:265-6.

32. Shaper A, Wannamethee SG, Walker M. Pipe and cigar smoking and major cardiovascular events, cancer incidence and all-cause mortality in middle-aged British men. Int $\mathrm{J}$ Epidemiol. 2003;32:802-8.

33. Evans J, Hyndman S, Stewart-Brown S, Smith D, Petersen S. An epidemiological study of the relative importance of damp housing in relation to adult health. $\mathrm{J}$ Epidemiol Community Health. 2000;54:677-86.

34. Rennie D, Chen Y, Lawson J, Dosman J. Differential effect of damp housing on respiratory health in women. JAMA. 2005;60:46-51.

35. Kilpeläinen $\mathbf{M}$, Terho $\mathbf{E}$, Helenius $\mathbf{H}$, Koskenvuo $\mathbf{M}$. Home dampness, current allergic diseases, and respiratory infections among young adults. Thorax. 2001;56:462-7.

36. Gunnbjörnsdóttir M, Franklin K, Norbäck D, Björnsson E, Gislason D, Lindberg E, et al. Prevalence and incidence of respiratory symptoms in relation to indoor dampness: The RHINE Study. Thorax. 2006;61;221-5.

37. Welle I, Eide G.E, Gulsvik A, Bakke PS. Pulmonary gas exchange and educational level: A community study. Eur Respir J. 2004;23:583-8.

38. Martins M, Fatigati FL, Véspoli TC, Martins LC, Pereira LA, Martins MA, et al. Influence of socioeconomic conditions on air pollution adverse health effects in elderly people: An analysis of six regions in São Paulo, Brazil. J Epidemiol Community Health. 2004;58;41-6.

39. Prescott E, Lange P, Vestbo J. Socioeconomic status, lung function and admission to hospital for COPD: Results from the Copenhagen City Heart Study. Eur Respir J. 1999;13:1109-114 
40. Jerrett M, Burnett T, Brook J, Kanaroglou P, Giovis $\mathrm{C}$, Filkestein $\mathbf{N}$, et al. Do socioeconomic characteristics modify the short term association between air pollution and mortality? Evidence from a zonal time series in Hamilton, Canada. J Epidemiol and Community Health. 2004;58:31-40.

41. Medina-Ramón M, Zock JP, Kogevinas M, Sunyer J, Antó JM. Asthma symptoms in women employed in domestic cleaning: A community based study. Thorax. 2003;58:950-4

42. Matheson MC, Benke G, Raven J, Sim MR, Kromhout $\mathbf{H}$, Vermeulen $\mathbf{R}$, et al. Biological dust exposure in the workplace is a risk factor for chronic obstructive pulmonary disease. Thorax. 2005;60:645-51.

43. Cançado J, Saldiva P, Pereira L, Lara L, Artaxo P, Martinelli $\mathrm{L}$, et al. The impact of sugar cane-burning emissions on the respiratory system of children and the elderly. Environ Health Perspect. 2006;114:725-9.

44. Abbey D, Burchette R, Knutsen S, Mcdonnell W, Lebowitz M, Enright P. Long-term particulate and other air pollutants and lung function in nonsmokers. Am $\mathrm{J}$ Respir Crit Care Med. 1998;158:289-98.

45. Shohaimi S, Welch A, Bingham S, Luben R, Day N, Wareham $\mathbf{N}$, et al. Area deprivation predicts lung function independently of education and social class. Eur Respir J. 2004;24:157-61.

46. Alfonso HS, Fritschi L, Klerk NH, Olsen N, Sleith J, Musk A. Effects of asbestos and smoking on the levels and rates of change of lung function in a crocidolite exposed cohort in Western Australia. Thorax. 2004;59:1052-6.

47. Beeckman L, Wang M, Petsonk E, Wagner G. Rapid declines in $\mathrm{FEV}_{1}$ and subsequent respiratory symptoms, illnesses, and mortality in coal miners in the united states. Am J Respir Crit Care Med. 2001;163:633-9.

48. Bergdahl I, Tore'n K, Eriksson K, Hedlund U, Nilssonz $\mathbf{T}$, Flodin $\mathbf{R}$, et al. Increased mortality in COPD among construction workers exposed to inorganic dust. Eur Respir J. 2004;23:402-6 